Bull. Austral. Math. Soc.

VOL. 56 (1997) [439-445]

\title{
SMOOTHNESS OF THE SOLUTION OF THE TRIVIAL MONGE-AMPERE EQUATION
}

\author{
VITALY USHAKOV
}

\begin{abstract}
A solution of the equation $z_{x x} z_{y y}-z_{x y}^{2}=0$ must be $C^{2}$ smooth, need not be $C^{3}$ smooth, but at the same time the ratio $z_{x y}: z_{x x}$ is $C^{1}$ (provided $z_{x x} \neq 0$ ). An analytical proof of this fact is given; the underlying geometrical interpretation is discussed.
\end{abstract}

\section{BASIC DEFINITIONS AND STATEMENTS}

The trivial Monge-Ampère equation is

$$
\operatorname{Hess}(z)=z_{x x} z_{y y}-z_{x y}^{2}=0
$$

and sometimes is called the equation of developable surfaces $[\mathbf{1}, \mathrm{p} .10]$.

Theorem 1. Let a $C^{2}$ smooth function $z: D \subset \mathbb{R}^{2} \rightarrow \mathbb{R}$ be a solution of the equation Hess $(z)=0$ and assume $z_{x x} \neq 0$. Then $z_{x y}: z_{x x}$ is $C^{1}$ smooth.

REMARK 1. The assumptions Hess $(z)=0$ and $z_{x x} \neq 0$ could be replaced with

$$
\operatorname{rank}\left(\begin{array}{cc}
z_{x x} & z_{x y} \\
z_{y x} & z_{y y}
\end{array}\right)=1
$$

then the conclusion is $z_{x y}: z_{x x}=z_{y y}: z_{x y} \in C^{1}$.

REMARK 2. Theorem 1 is precise in the following sense: $z \in C^{2}$ but need not be $C^{3}$ smooth; the quotient $z_{x y}: z_{x x}$ is $C^{1}$, but need not be $C^{2}[8]$.

Theorem 1 although having a purely analytic formulation, possesses a sound geometric interpretation. If one considers the surface $F \subset E^{3}$ with the radius vector $(x, y, z(x, y))$ given over the domain $D \subset \mathbb{R}^{2}$ then the condition Hess $(z)=0$ means the vanishing of the Gaussian curvature; the condition $z_{x x} \neq 0$ guarantees the absence of planar points (that is, points with $z_{x x}=z_{x y}=z_{y y}=0$ ). These conditions provide a linear structure for the surface $F$ and, moreover, the tangent plane of $F$ is stable

Received 14th January, 1997.

I would like to thank Professor H. Rubinstein and Professor W. Neumann who kindly afforded me an opportunity to work at the University of Melbourne.

Copyright Clearance Centre, Inc. Serial-fee code: 0004-9729/97 \$A2.00+0.00. 
along these rectilinear generators. (A linear surface with such a property is called a torse, following Euler.) In other words there exists a linear parametrisation $\widetilde{r}(u, v)$ of the surface $F$ such that

$$
r(x, y)=\left(\begin{array}{c}
x \\
y \\
z(x, y)
\end{array}\right)=\widetilde{r}(u, v)=a(u)+v \cdot b(u), \quad\|b(u)\|=1
$$

where $a(u)$ sets a directrix, $b(u)$ gives the direction of the generators; the condition of stability of $T_{\tilde{r}(u, v)} F=\operatorname{span}\{\dot{a}+v \dot{b}, b\}$ means the independence of $T F$ from the parameter $v$, that is, $\dot{b} \in \operatorname{span}\{\dot{a}, b\}$.

But what is the smoothness of $a(u)$ and $b(u)$ ? Can we differentiate them (in the hypothesis of $C^{2}$ smoothness of the surface $F$, that is, $\left.z(x, y) \in C^{2}\right)$ ?

The function $a(u)$ being almost an arbitrary curve on the surface $F$ (we need only the transversality with the generators) can be easily chosen $C^{2}$ smooth. But the direction $b(u)$ is determined uniquely. It turns out that the quotient $z_{x y}: z_{x x}$ from Theorem 1 describes precisely the direction of $b(u)$. Indeed, at the point $(x, y, z(x, y))$ the vector $b(u)$ annihilates the second fundamental form of the surface, that is, for $b(u)=b_{1}(u) r_{x}+b_{2}(u) r_{y}$ we have

$$
\left(\begin{array}{ll}
z_{x x} & z_{x y} \\
z_{y x} & z_{y y}
\end{array}\right)\left(\begin{array}{l}
b_{1} \\
b_{2}
\end{array}\right)=0
$$

hence $b_{1}(u): b_{2}(u)=-z_{x y}: z_{x x}$. Finally, the geometric reformulation of Theorem 1 is as follows.

THEOREM 2. Let a $C^{2}$ smooth surface $F \subset E^{3}$ given over a simply connected domain $D \subset \mathbb{R}^{2}$ possess vanishing Gaussian curvature and assume it does not contain planar points. Then

(1) $F$ is a torse;

(2) the vector field setting the directions of the generators is $C^{1}$ smooth.

REMARK. The statement $b(u) \in C^{1}$ is not obvious. For instance, if one omits the condition on the absence of planar points (while the vector field $b(u)$ may be well defined: a point $u_{0}$ is planar and at the same time is a cluster point for non-planar ones) then $b(u) \in C^{0}$ and need not be $C^{1}[7]$.

In this form Theorem 2 was stated in the work [3, Corollary 3] with references in the proof to [4, Theorem 5] and [5, Theorem 14]. Therefore, we can not claim Theorem 1 to be new. Nevertheless, the proof of Theorem 2 is scattered over three different papers and inside the papers by preparatory stages. Besides that in places the proof lacks clearness. As a result, the conclusion about smoothness in Theorem 2 is little-known 
and the public is primarily aware of conclusion (1) only. At the same time this result is basic in the theory of surfaces of constant nullity and Riemannian manifolds of constant nullity. It has been generalised with respect to dimension and codimension (the latter, of course, for surfaces); see [6] for references. These facts as well as the possibility of a purely analytic formulation and proof compelled us to the present note. In Section 2 we prove Theorem 1 and then in Section 3 pass to the geometric interpretation of the steps.

\section{ProOFS}

The proof of Theorem 1 is based on the following Lemma.

LEMma 1. In the hypotheses of Theorem 1 the domain $D$ is fibred on rectilinear generators along which the function $z_{x}$ is constant.

Lemma 1 is a direct corollary of the following fact.

Lemma 2. Let a curve $y=y(x)$ be a level curve of the function $z_{x}(x, y)$ where $z: D \subset \mathbb{R}^{2} \rightarrow \mathbb{R}$ is $C^{2}$ smooth and $z_{x x} \neq 0$. Then $\ddot{y}(x)$ exists if and only if the partial derivative (Hess $\left.(z) / z_{x x}\right)_{x}$ exists; $\ddot{y}$ can be computed by the formula

$$
\ddot{y}=-\frac{z_{x x}^{2}}{z_{x y}^{3}}\left(\frac{\operatorname{Hess}(z)}{z_{x x}}\right)_{x} ;
$$

the actual curvature of $y(x)$ is given by

$$
k=-\frac{z_{x x}^{2}}{\left(z_{x x}^{2}+z_{x y}^{2}\right)^{3 / 2}}\left(\frac{\operatorname{Hess}(z)}{z_{x x}}\right)_{x} .
$$

In the proof of Lemma 2 we shall use the following.

PROPOSITION. Let a curve $y=y(x)$ be a level curve of a $C^{2}$ smooth function $\phi: D \subset \mathbb{R}^{2} \rightarrow \mathbb{R}$. Then $\ddot{y}$ is given by the formula

$$
\ddot{y}=-\frac{1}{\phi_{y}^{3}}\left[\phi_{x x} \phi_{y}^{2}-2 \phi_{x y} \phi_{x} \phi_{y}+\phi_{y y} \phi_{x}^{2}\right] .
$$

Proof of Proposition: Since $y=y(x)$ sets a level curve of the function $\phi$, we have

$$
\phi(x, y(x)) \equiv \text { const } .
$$

Differentiating this equation with respect to $x$, we obtain $\phi_{x}+\phi_{y} \cdot \dot{y}=0$, hence

$$
\dot{y}=-\frac{\phi_{x}}{\phi_{y}} .
$$


Further,

$$
\begin{aligned}
& \ddot{y}=-\left(\frac{\phi_{x}}{\phi_{y}}(x, y(x))\right)_{x}=-\frac{\left(\phi_{x x}+\phi_{x y} \cdot \dot{y}\right) \cdot \phi_{y}-\phi_{x} \cdot\left(\phi_{y x}+\phi_{y y} \cdot \dot{y}\right)}{\phi_{y}^{2}} \\
& \stackrel{(5)}{=}-\phi_{y}^{-3}\left[\phi_{x x} \phi_{y}^{2}-2 \phi_{x y} \phi_{x} \phi_{y}+\phi_{y y} \phi_{x}^{2}\right] .
\end{aligned}
$$

Proof of Lemma 2: Let us assume for the beginning that $z \in C^{3}$ and later discuss how to drop this assumption. Direct application of the Proposition to $\phi=z_{x}$ gives

$$
\begin{aligned}
\ddot{y} & =-\frac{1}{z_{x y}^{3}}\left[z_{x x x} z_{x y}^{2}-2 z_{x x y} z_{x x} z_{x y}+z_{x y y} z_{x x}^{2}\right] \\
& =-\frac{1}{z_{x y}^{3}}\left[z_{x x x}\left(z_{x x} z_{y y}-\operatorname{Hess} z\right)-2 z_{x x y} z_{x x} z_{x y}+z_{x y y} z_{x x}^{2}\right] \\
& =-\frac{1}{z_{x y}^{3}}\left[z_{x x}\left(z_{x x x} z_{y y}+z_{x x} z_{y y x}-2 z_{x y} z_{x x y}\right)-z_{x x x} \operatorname{Hess} z\right] \\
& =-\frac{1}{z_{x y}^{3}}\left[z_{x x}(\operatorname{Hess} z)_{x}-z_{x x x} \operatorname{Hess} z\right]=-\frac{z_{x x}^{2}}{z_{x y}^{3}}\left(\frac{\operatorname{Hess} z}{z_{x x}}\right)_{x}
\end{aligned}
$$

which is the desired formula (3). To obtain the curvature it is sufficient to apply the standard expression $k=\ddot{y}\left(1+\dot{y}^{2}\right)^{3 / 2}$.

The formula (3) for the case $z \in C^{2}$ can be derived in the same way, but now we should avoid the third partial derivatives $z_{x x x}$, et cetera. That is possible if we leave everything under the limit sign up to the final step:

$$
\begin{aligned}
\ddot{y} & \stackrel{(5)}{=}-\left(\frac{z_{x x}}{z_{x y}}(x, y(x))\right)_{x} \\
& =-\lim _{\varepsilon \rightarrow 0}\left[\frac{z_{x x}}{z_{x y}}(x+\varepsilon, y(x+\varepsilon))-\frac{z_{x x}}{z_{x y}}(x, y(x))\right] \\
& =\cdots=z_{x y}^{-3} \lim _{\varepsilon \rightarrow 0}\left[z_{x x} \cdot\{\operatorname{Hess} z(x+\varepsilon, y)-\operatorname{Hess} z(x, y)\}\right. \\
& =-\frac{z_{x x}^{2}}{z_{x y}^{3}}\left(\frac{\operatorname{Hess} z}{z_{x x}}\right)_{x} .
\end{aligned}
$$

The step omitted is a simple though tiresome technical exercise.

Proof of Theorem 1: By applying Lemma 1 we obtain the fibration of the domain $D$ on rectilinear generators along which $z_{x}$ is constant. The normal vector to such a generator at $\left(x_{0}, y_{0}\right)$ is $\left(z_{x x}\left(x_{0}, y_{0}\right), z_{x y}\left(x_{0}, y_{0}\right)\right)$ and since this direction is invariable on the generator, $z_{x y}: z_{x x}$ is constant on the generator. The condition $z_{x x} \neq 0$ implies the transversality of the generators to the $x$-axis and therefore the 
general statement $z_{x y} / z_{x x}(x, y) \in C^{1}$ can be reduced to $z_{x y} / z_{x x}(x, 0) \in C^{1}$, that is, now we need to prove the statement along the $x$-axis only.

The latter fact is easily derived from the following identity:

$$
z_{x}\left(x-\frac{z_{x y}}{z_{x x}}(x, 0), 1\right)=z_{x}(x, 0) .
$$

Indeed, the function $\psi(\xi)=z_{x}(\xi, 1)$ is $C^{1}$ and is invertible $\left(z_{x x}(x, 1) \neq 0\right)$; therefore $\psi^{-1} \in C^{1}$ and because of $(6)$

$$
\frac{z_{x y}}{z_{x x}}(x, 0)=x-\psi^{-1}\left(z_{x}(x, 0)\right) \in C^{1} .
$$

The identity (6) itself simply reflects the fact of constancy of $z_{x}$ along a generator: the generator through $\left(x_{0}, 0\right)$ has normal $\left(z_{x x}\left(x_{0}, 0\right), z_{x y}\left(x_{0}, 0\right)\right)$ and therefore passes through the point $\left(x_{0}-\left(z_{x y} / z_{x x}\right)\left(x_{0}, 0\right), 1\right)$.

\section{Geometric parallels}

In this section we outline the geometric proof of Theorem 2 (of Hartman and Nirenberg [3]) comparing it at the same time with our proof of Theorem 1.

First temptation. Considering Theorem 2 from a geometrical point of view it would be natural to single out at each point the asymptotic direction (which is $d x: d y=$ $\left.-z_{x y}: z_{x x}\right)$ and find the integral lines of that distribution. For $C^{2}$ smooth function $z$ it leads to the differential equation

$$
\frac{d y}{d x}=-\frac{z_{x x}}{z_{x y}}(x, y)
$$

the right hand side of which is only continuous and hence we can not even claim the existence and uniqueness of the integral lines. (One needs the right hand side to be Lipschitzian in $y$.)

First INTEGRALS. Therefore, following Hadamard [2, pp.398-399] (who probably in turn followed Darboux) we notice that the differential equation (7) possesses a first integral-the function $z_{x}$ (that is, $z_{x}$ is constant along the solution of (7)). Besides that the function $z_{y}$ is a first integral as well. Indeed,

$$
\begin{aligned}
& d_{(d x, d y)} z_{x}=z_{x x} \cdot d x+z_{x y} \cdot d y=0 \quad(\text { by (7)), } \\
& d_{(d x, d y)} z_{y}=z_{y x} \cdot d x+z_{y y} \cdot d y=0 \quad \text { (by (1)). }
\end{aligned}
$$

Thereby the study of asymptotic lines given by the equation (7) has been reduced to a more pleasant problem of studying the level curves of the function $z_{x}$. Note, the condition $z_{x x} \neq 0$ guarantees the absence of singularities on these curves. 
ThE STABILITY OF TANGENT PLANE ALONG THE Lines $z_{x}=$ const. The condition $z_{x}=$ const means the constancy of the basis tangent vector $r_{x}=\left(1,0, z_{x}\right)$ in the asymptotic direction. The constancy of both basis tangent vectors $r_{x}$ and $r_{y}$ means the stability of the tangent plane (and then the normal) in these directions. Consequently the lines $z_{x}=$ const possess vanishing normal curvature. Moreover, the tangent plane at the point $\left(x_{0}, y_{0}\right)$ is tangent to the surface $F$ along the whole line $z_{x}=z_{x}\left(x_{0}, y_{0}\right)=$ const.

The surface $F$ is the envelope of a 1-PARAmeter family of Planes. These planes can be obtained as the tangent planes to $F$ along an arbitrary curve on $F$ which is transversal to the lines $z_{x}=$ const.

The LeVel CURVES OF THE FUnCTION $z_{x}$ POSSESS VANISHING geodesic curvature. This is the most technically demanding step of the proof. We used here Lemma 1 while Hadamard and his successors employed the theorem of the envelope of 1 parameter family of planes [4, p.770]. The geometric picture is as follows: two infinitesimally close planes from the family must intersect along a curve lying on the surface; but the line of intersection of two planes is straight.

Change of variables. Now having proved the level curves of $z_{x}$ are straight lines, we develop the following $C^{1}$ smooth change of variables:

$$
\left\{\begin{array}{l}
u=z_{x}(x, y) \\
v=y
\end{array}\right.
$$

The new radius vector $\widetilde{r}(u, v)=r(x(u, v), y(u, v))$ is $C^{1}$ smooth and for a fixed $u=$ $u_{0}$ the radius vector $\widetilde{r}\left(u_{0}, v\right)$ draws a generator. A vector field $b(u)$ which gives the direction of these generators can be chosen as $b(u)=r(u, 1)-r(u, 0)$, which is $C^{1}$ smooth. In our proof this step corresponds to the identity (6).

\section{REFERENCES}

[1] R. Courant and D. Hilbert, Methods of mathematical physics II, Partial differential equations (Interscience publishers, New York, 1962).

[2] J. Hadamard, Cours d'analyse I (Herman, Paris, 1927).

[3] P. Hartman and L. Nirenberg, 'On spherical image maps whose Jacobians do not change sign', Amer. J. Math. 81 (1959), 901-920.

[4] P. Hartman and A. Wintner, 'On the fundamental equations of differential geometry', Amer. J. Math. 72 (1950), 757-774.

[5] P. Hartman and A. Wintner, 'On the asymptotic curves of a surface', Amer. J. Math. 73 (1951), 149-172. 
[6] V. Ushakov, Riemannian manifolds and surfaces of constant nullity (in Russian), Thesis (St. Petersburg University, 1993).

[7] V. Ushakov, 'Parametrization of developable surfaces by asymptotic lines', Bull. Austral. Math. Soc. 54 (1996), 411-421.

[8] V. Ushakov, 'The explicit general solution of trivial Monge-Ampère equation', (preprint, University of Melbourne, 1996).

Department of Mathematics

The University of Melbourne

Parkville Vic 3052

Australia

e-mail: vit@mundoe.maths.mu.oz.au 